\title{
Research on the Cross-Platform Communication Technologies and the Corresponding Usages in Smart Home Systems
}

\author{
Wenzhun Huang ${ }^{1, a^{*}}$, Xinxin Xie ${ }^{1, b}$ and Shanwen Zhang ${ }^{1, c}$ \\ ${ }^{1}$ Department of Electronic Information Engineering, Xijing University, Xi'an 710123, China

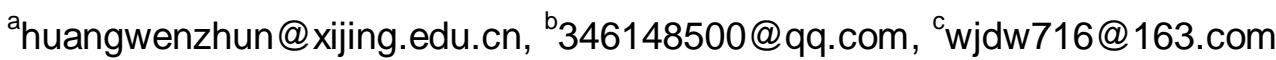

Keywords: Cross-Platform communication; Smart home; Applications; Datat transformation.

\begin{abstract}
In this paper, we conduct research on the cross-platform communication technologies and the corresponding usages in smart home systems. Along with the rapid development of society, the human common higher demands on household environment, pay more attention to the convenience of household environment, convenience, comfort and intelligent, intelligent household concept comes from the Internet of things, through the Internet of things technology will be the family household electrical appliances, instruments and meters, lighting, communication equipment, basic monitoring equipment connected to the Internet, through the remote to control related operations, so as to realize intelligent home control system. Under this historical era we enhance the basic traditional smart home with the enhancement of the cross-platform communication that is innovative.
\end{abstract}

\section{Introduction}

Smart home is to use the advanced network communication technology, automatic control technology and computer technology, will be related with the household life of all sorts of household equipment organically unifies in together, through the network of integrated management, let household life more easily. With all kinds of the new technology constantly emerging and smart home is also in the continuous development of the dynamic change [1-2].

According to the review, the types of the smart home systems could be separated into the following parts. (1) Centralized control technology. In smart home system, if uses the basic centralized control technology, the single chip computer is indispensable, it is the main part of the signal processing, and some peripheral circuit such as communication circuit, alarm circuit and cooperation. (2) The fieldbus technology. For fieldbus intelligent household system of network topology is not has a fixed structure, bus is the communication distance, the module connected to the bus in the network can communicate, and this simplifies wiring in the very great degree that expands the general capacity. (3) Power carrier technology. Another technology for smart home system is the high frequency power carrier as it is the key technology of the application of wireless technology. System, the high frequency signal makes the transmission of information. Send the launcher launched high frequency signal by the information and then through the power line transmission, eventually be received at the receiver end, such a process to reach the control purpose and the corresponding scenarios [3].

Smart home control system has become a variety of the related technologies, including the sensors, communications, automatic control, database, security, audio and general video technology, sensor technology is used to detect smart home control system within the surrounding environment, as the communication technology in smart home control system internal device for network connection and mutual communication, basic automatic control technology used in intelligent home control system to control the equipment and operation, the database used to store the relevant data of the control system of the smart home information and the key technologies to implement the current smart home network control system for compatibility strong family main controller and the family network can meet the needs of information transmission, with the PC architecture, architecture, SCM (single chip micyoco embedded architecture three solutions have cable technology, wireless technology, the electric power carrier technology, etc. as the main way of the three major categories. 
In the intelligent home network, whether it's personal consumption or collective for application, the user's demand in most case, it is reflected in the application of specific solutions, only solution was put forward and the application, the user's actual needs will be met and a lot of problems are solved. As the illustration, we show the sample smart home systems user management platform as follows.

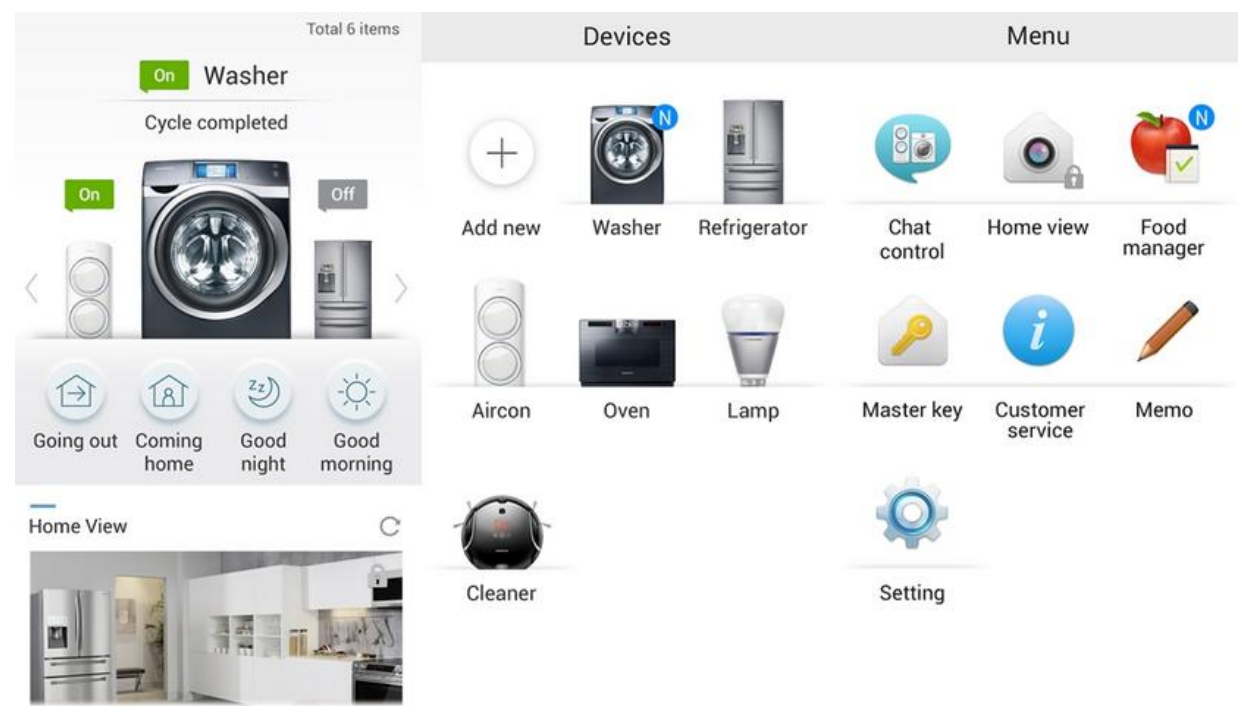

Figure 1. The Sample Smart Home Systems Unser Management Platform

In this paper, we conduct theoretical research on the cross-platform communication technologies and the corresponding usages in smart home systems. It is the development trend of the smart home network system varies according to the requirement of the use of group, now use group is more and more hope that the use of the product function toward the more powerful, more intelligent direction, and at the same time of operation is simple, more want to consider the concept of human nature. More importantly, the cross-platform communication has been the developmental trend.

\section{The Proposed Method}

The Review of the Smart Home Systems. The so-called smart home is on the basis of residential, plan as a whole management in all aspects of family life, scientific design from the different aspects to improve the household life of high quality, and eventually making into household life "wisdom". In today's era, the Internet of things technology has the electronic commerce in our country, the hospital management, education informatization construction, urban construction, environmental protection and so on many areas have become more widely used.

Based on embedded technology, designed a kind of intelligent home control system and the system can better realize the indoor temperature monitoring, gas monitoring, lighting monitoring, entrance guard monitoring, and other functions that can be listed as the follows.

1 Wireless RF module. Home server first to establish a socket, and then has been in the listening state, waiting for the dashboard connection request, when the dashboard, after sending the connection request to the home server, with the server receives the request, and establish a receiving threads handle communication with the dashboard [4-5].

2 Power supply module. Power supply module is mainly used for basic embedded intelligent household system of energy supply embedded intelligent household system to run normally, each subsystem is to be without power supply module power supply, power supply module through the way of the cable to connect with each device.

3 The sensor. Sensor DS18B20 temperature monitoring, gas monitoring sensor MQ-2 infrared detector and the human body that is used for intelligent household environment monitoring information, the sensors by wireless RF module with the I/O interface connection. 
No operating system through the infinite loop continuously to monitor events, data extraction, but only shows the final data values, and the operating system is in constant to monitor events, the process of data extraction can undertake interruption at any time, and then achieve the corresponding control function with the realization of the embedded intelligent home control system all functions, all the control and operation of equipment that all from the background software monitoring platform.

The Communication Technologies for the Smart Home Devices. It has launched on the market at present intelligent household category of various products that can provide different services.

From the point of view of application system, installed in the interior of all kinds of product is the largely composed of terminal nodes and general control nodes, some of control node corresponding equipment function more concentrated, can communicate with the terminal nodes as can also with the external network unicom, such as the IPTV set-top box and some control node functions provided by the professional control device and the gateway to respectively.

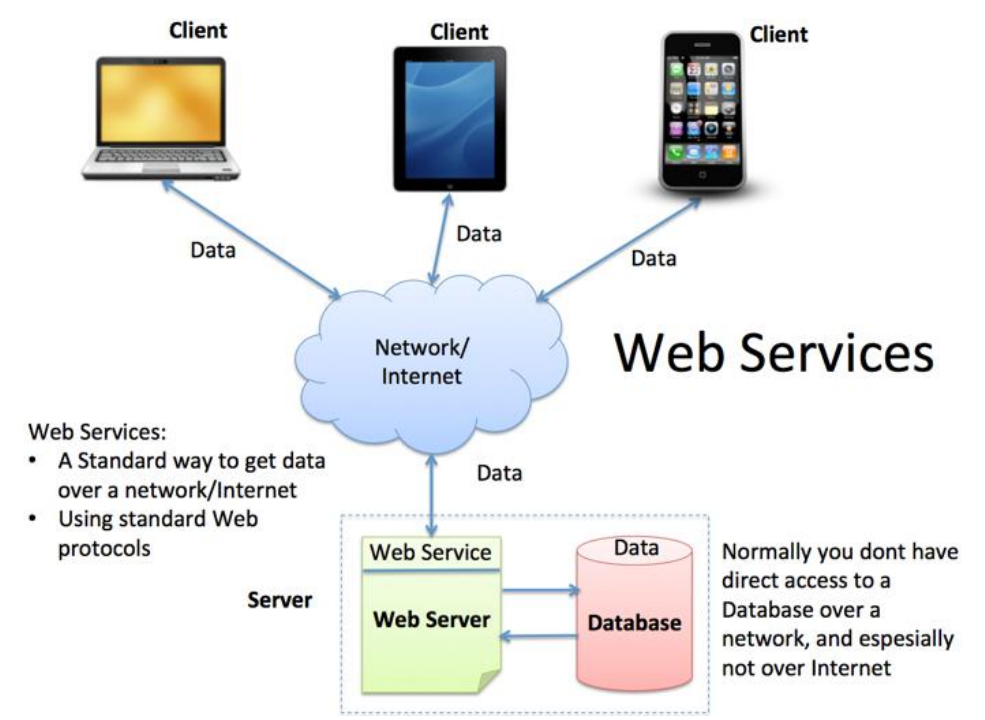

Figure 2. The Communication Technologies for the Smart Home Devices

Control information and the multimedia information can be transmitted through wired or wireless communication way, compared with wireless communication mode, the cable communication mode of smart home system in scalability, mobility, and degree of degree of wiring and trival that beautiful slightly weak, combined with the characteristics of the smart home.

1 Infrared communication technology is a point to point data transmission protocol, is one of the traditional equipment connection between cable replacement, it generally within one meter, communication distance communication medium for near infrared infrared wavelengths. But infrared transmission distance is short, poor permeability of opaque objects, not through the wall, unable to use the technology to the indoor other products send the remote signal of the whole room, so the technology is only suitable for the super close transmit control information and the multimedia information [6].

2 Zigbee technology is a set of based on the IEEE802.15.4 wireless standards developed by the networking, security and general application of the communication technology its outstanding characteristic is close, low complexity and low power consumption, low data rate, low cost, have ad-hoc network and the recovery ability with a single network can support 255 devices.

3 Bluetooth technology is mainly used in computers, mobile phones, printers, keyboards, mouse and other equipment in the absence of cable connection to communicate with each other, or wireless Internet sharing. Due to the cost of the blue-tooth high all the time, it also become a stumbling block of the application of large-scale household control in the future.

4 RFID technology can be used for the induction of a moving object within a, such as static objects access to information, communication distance can be up to 45 meters, as far as the data transfer 
rate and length of the RFID tag information as encoding and request to read the label every second times.

The Cross-Platform Communication. For mobile terminal software and hardware resources of the network operating system needs to be unified management network, for the application of the end user to provide convenient and effective services. In the mobile cloud computing, various examples operating system as a kind of software resources for basic unified management and scheduling as the function of the new network operating system deployed in mobile terminal and server, so as to build up a distributed with hierarchical network operating system architecture supports dynamic loading.

On the server side, the deployment of the multiple instance of the operating system update list, data management, user management monitoring the server operating system run time server-side real-time response to the mobile terminal of the interrupt request service, and according to service requirements of the transmission response resources. The entire platform architecture implements the user anytime, anywhere access needed for the various application service, meet the demand of the application software for the platform, the difference of the also solved the limitations of mobile terminal resource constraints. The following figure shows the sample code blocks [7].

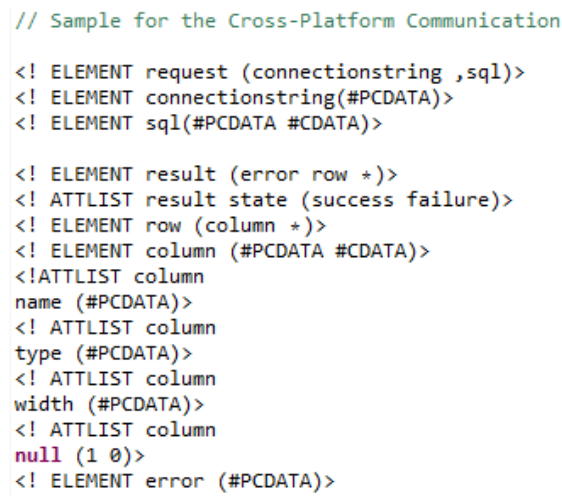

Figure 3. The Sample XML Code for the Cross-Platform Communication

Windows client service process using the multi-thread mechanism, that not too many connections, otherwise it will lead to the sharp decline in performance of the system. For reasons of data query efficiency, every time do not want to submit this request immediately connect the database instance destructor, but continue to maintain a constant connection state, if the user request again within the specified time interval, then use the same database connection instance that rather than to build a new connection instance. The software in order to make all kinds of storage on the server can perform at the mobile terminal we can design a network interruption technology, dynamic scheduling instances operating system instructions and data to the network terminal through the basic network interrupt processing for interruption from virtual device. If the virtual device interrupt on the mobile terminal, the interruption of transmission through the network to the server side, after processing by the server through the network to process the results back to the mobile terminal [8].

In order to make the middle tier can platform-independent, used the Java technology, as a service client and uses the pure Java language programming interface. Application server uses multi-thread technology to implement multiple request processing and using JDBC, ODBC database connection. For reasons of portability, use the Java language programming interface, it provides the programming interface can be invoked for web-based applications that is typical Java application, Servlet, applets.

Response between the two sides in accordance with the contract, the client to the server to send data update request, we can choose the time or manually. The client use the relevant application will accept the new fields of data updating corresponding to the local database table. The client data query in the local, the whole process of data synchronization is transparent to the user, the user feels like when complete data query to remote database and reach both local and remote data synchronization 


\section{Conclusions}

In this paper, we conduct theoretical research on the cross-platform communication technologies and the corresponding usages in smart home systems. Because of the cloud to the basic shared resource heterogeneity, how to use these Shared resources across platforms is currently an urgent need to solve the problem. In intelligent mobile terminal cross-platform application study, solve the heterogeneous network access, examples of the operating system of the core problems of loading, a cross-platform system design method based on cloud computing. Numerical simulation shows that the performance according to the general pattern design of algorithm of cross-platform middleware on the premise of guarantee calculation precision greatly improve the processing time, for the information processing of embedded software parallelization design and optimization provides a new way of thinking. In recent future, we will conduct the hardware implementation to test the efficiency.

\section{References}

[1] N. K. Suryadevara, S. C. Mukhopadhyay, R. Wang and R. K. Rayudu: Engineering Applications of Artificial Intelligence, Vol. 26 (2013) No.10, p.2641.

[2] J. D. Logue, S. Supramaniam, O. Hardison and J. Luxenberg: U.S. Patent No. 9,237,141. 12 Jan. 2016.

[3] M. Soliman, T. Abiodun, T. Hamouda and J. H. Zhou: 2013 IEEE 5th International Conference on Cloud Computing Technology and Science (Bristol, Dec. 2-5, 2013). Vol. 2, p.317.

[4] S. C. Kim, Y. S. Jeong and S. O. Park: Personal and ubiquitous computing, Vol. 17 (2013) No.8, p.1699.

[5] M. Theoharidou, N. Tsalis and D. Gritzalis: Handbook of smart homes, health care and well-being (2014).

[6] Y. L. Hsueh, N. H. Lin, C. C. Chang and O. T. C. Chen: 2015 8th International Conference on Ubi-Media Computing (Colombo, Aug. 24-26, 2015), p. 273.

[7] M. Vacher, A. Fleury, F. Portet and N. Noury: Digital Advances in Medicine, E-Health, and Communication Technologies (2013), p.148.

[8] Md. Sumon Shahriar, and M. Sabbir Rahman: Proceedings of the 2015 International Workshop on Internet of Things towards Applications (ACM New York, NY, USA, 2015), p.19. 